\title{
Pseudoangiomatous Stromal Hyperplasia: Diagnosis, Treatment and Follow-Up; Description of a Case-Series
}

\author{
W.A. Donk ${ }^{*}$, R.J. Oostenbroek, R.K. Storm, P.J. Westenend and P.W. Plaisier \\ Department of Surgery, Albert Schweitzer hospital, P.O. Box 444, 3300 AK, Dordrecht, The Netherlands
}

\begin{abstract}
Pseudoangiomatous stromal hyperplasia (PASH) is a benign lesion of the breast. Mammography and ultrasonography usually reveal a well circumscribed lesion with benign characteristics. In this report a series of 36 patients is described in terms of imaging, histopathologic findings, therapy and follow- up. Also an overview of the literature is provided. No standard excision is necessary for PASH proven by core needle biopsy. However, it should be noted that PASH may be an incidental finding not representative of the targeted lesion.
\end{abstract}

Keywords: Pseudoangiomatous stromal hyperplasia (PASH), Mammography, ultrasonography, imaging, histopathologic findings.

\section{INTRODUCTION}

Pseudoangiomatous stromal hyperplasia (PASH) is a benign lesion of the breast, first described by Vuitch et al. in 1986 [1]. Women with this disorder usually present with a painless palpable mass in the breast, or a suspicious lesion on screening mammography. Over $50 \%$ of women with PASH is pre-menopausal. PASH is often an incidental finding alongside a benign or malignant tumor. Tumors that entirely consist of PASH are rare. A diagnosis of PASH can be confidently made on a core needle biopsy but recommendations on the treatment and follow-up are somewhat controversial. Especially in the nineties complete excision of the lesion was advocated because of the uncertainty concerning the biological behavior of PASH. Another point of consideration is the possibility of a sampling error. In this article we describe the diagnosis, treatment and follow-up of a series of patients diagnosed with PASH.

\section{METHODS}

All patients diagnosed with PASH in our hospital in the period january 2000 - august 2010 , either by core-needle biopsy or excisional biopsy, were identified from the database of our pathology laboratory. Of these 36 patients, the clinical records were retrieved and characteristics were collected retrospectively. Age, history of breast abnormalities, use of oral contraceptives or hormone replacement therapy and family history were scored as was the way how patients presented to our hospital. Results of all diagnostic procedures performed (mammography, ultrasonography, fine needle aspiration [FNA], core needle biopsy and excisional biopsy) were noted as well as the follow up performed. For radiological diagnostics the BIRADS $^{\circledR}$ classification was used [2]. All mammographies and ultrasonographies were revised by an experienced

*Address correspondence to this author at the Department of Surgery, Albert Schweitzer hospital, P.O. Box 444, 3300 AK, Dordrecht, The Netherlands; Tel: +31 78 6541111; Fax: +31 78 6179811;

E-mails:willemdonk@gmail.com,w.donk@asz.nl radiologist. The histological findings in patients with an inconsistency between the core-needle biopsy and the excisional biopsy were reviewed by our pathologist.

\section{RESULTS}

PASH was diagnosed in 36 patients ( 35 females, 1 male). Patient characteristics are described in Table 1. The majority of our patients $(61 \%)$ were pre-menopausal women, presenting with an asymptomatic palpable lump of the breast.

Table 1. Patient Characteristics of PASH

\begin{tabular}{|lc|}
\hline No. of Patients & 36 (35 Female; 1 Male) \\
Mean age (range) & $40(14-74)$ \\
History of breastpathology & \\
Yes & 9 \\
No & 19 \\
Unknown & 4 \\
Presentation & \\
Screening & 6 \\
On complaints & 30 \\
Complaints & \\
No & 5 \\
Palpable lump & 24 \\
Pain & 3 \\
Other & 5 \\
Duration(range) & \\
Hormonal status & 26 weeks (2 days - 3 years) \\
Pre-menopausal & \\
Post-menopausal & 22 \\
Unknown & 5 \\
Palpable lump & 9 \\
Yes & \\
No & 30 \\
\hline
\end{tabular}




\section{Imaging}

Results of imaging are described in Table 2. Mammography usually revealed a density, without microcalcifications or architectural distortion. In ultrasonography usually a solid, mixed echogenic lesion was found. Imaging was usually coded as BI-RADS III.

Table 2. Imaging Results of PASH

\begin{tabular}{|lc|}
\hline Mammography & 32 \\
Mass & 23 \\
Normal & 6 \\
Microcalcifications & 3 \\
Architectural distortion & 1 \\
Ultrasonography & 34 \\
Solid & 30 \\
Cystic & 0 \\
Mixed lesion & 0 \\
Hypoechoic & 5 \\
Mixed echogenicity & 21 \\
Hyperechoic & 3 \\
BI-RADS classification & \\
I & 0 \\
II & 0 \\
III & 26 \\
IV & 6 \\
V & 2 \\
\hline
\end{tabular}

\section{Pathological Findings}

PASH in core-needle biopsies or excisional biopsies was mainly described as clefts in collagen-rich tissue. The clefts were covered by endothelial-like cells, positive for CD34. Hormone receptors were not identified. Core needle biopsy or direct excision was preceded by FNA in 10 patients. PASH could not be diagnosed by FNA alone. Results of histopathology are summarized in Fig. (1). The results of histological examination of seven patients with inconsistent findings of core-needle biopsies and excisional biopsies were reviewed by a pathologist with experience in breast pathology. A summary is shown in Table 2. The inconsistency in patients $4,6,7$ was probably due to sampling errors. In patient 1 PASH was considered a codiagnosis with mastopathy as the main diagnosis. Patient 2 had a desmoid lesion with focal PASH after revision. We found one patient with two palpable masses in the left breast. Imaging revealed two lesions, compatible with a fibroadenoma. From both lesions material for pathologic study was obtained by core-needle biopsy. This revealed PASH in both biopsies. She had a lumpectomy at her own request. Pathologic study of these excisional biopsies revealed one lesion with PASH and the other invasive ductal carcinoma and ductal carcinoma in situ. Her choice of treatment was a mastectomy.

\section{Treatment and Follow-Up}

Sixteen patients (44\%) were treated by excisional biopsy. Two patients were treated with mastectomy for a BI-RADS 5 lesion with a high suspicion of malignancy. In one there was a mixed lesion in the core needle biopsy of PASH and a mesenchymal process; the other patient was treated for PASH accompanied with a carcinoma, as outlined before.

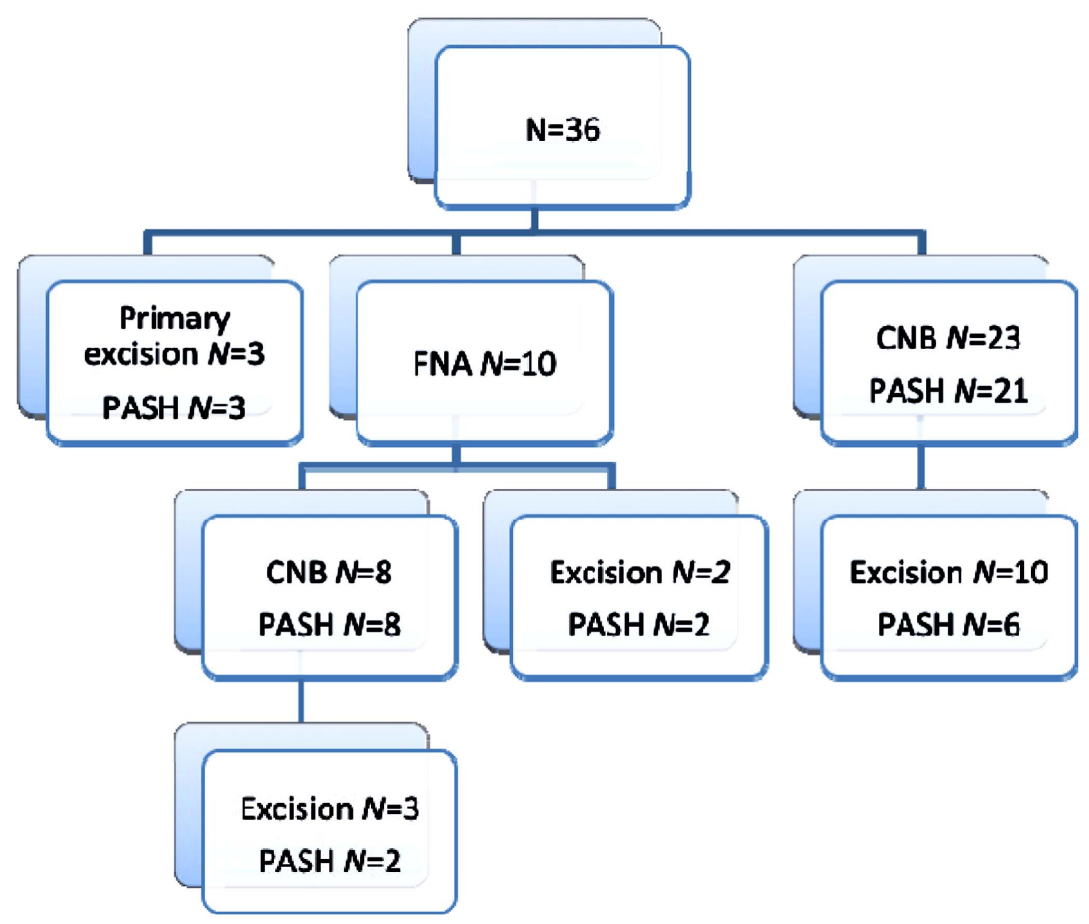

PASH: Pseudoangiomatous stromal hyperplasia; CNB Core Needle Biopsy; FNA Fine Needle Aspiration

Fig. (1). Results of histopathology. 
Eighteen patients were treated expectantly after the diagnosis of PASH (53\%).

Mean follow-up-time was 34 months (3-96). None of the patients developed breast cancer, seven patients developed additional, benign, lesions. According to PALGA, the nation-wide network and registry of histo- and cytopathology in the Netherlands none of the patients had a new FNA or core-needle biopsy in any other Dutch hospital for breast lesions during and after follow-up.

\section{DISCUSSION}

Since its first description in 1986, PASH has been the subject of several case reports and case-series. Most of the patients present with a palpable painless mass with regularly a circumscribed or focal lesion on the mammography (Fig. 2). When microcalcifications are also noted, a malignancy or a combination of malignancy and PASH has to be considered $^{3}$. In US most often a well-circumscribed hypo- or isoechogenic lesion is found, with a parallel orientation [3, 4] (Fig. 3). In our series $91 \%$ of the patients were identified with a solid lesion with a mean size of $4.3 \mathrm{~cm}$. It is well known that FNA is very unspecific, especially in diagnosing PASH. When performed, clustered intact bipolar spindleshaped mesenchymal cells may be found ${ }^{5,6}$. In 10 patients a
FNA was performed, all of them followed by core-needle biopsies or excisional biopsies. No explicit diagnosis could be made from FNA. The typical representation of open, slitlike anastomosing channels in stroma with a lining of myofibroblastic cells, with the channels lying in acellular, collagen-rich stroma $[5,6]$, was also described in pathologic reports in our clinic (Figs. 4, 5). In our pathology laboratory PASH was first described in 2000. Before then, it was usually described as a benign lesion without further classification. Obviously, immunohistochemical studies were used to support the diagnosis of PASH. The spindle cells express CD34, smooth muscle actin, vimetin and progesterone receptor, the latter suggesting an association of PASH and oral contraceptives, hormone replacement therapy and the strong reaction of PASH on tamoxifen as reported by Pruthi et al. in a case report [7, 8]. In our pathology laboratory, no routine progesterone receptor examination is performed in benign lesions. PASH must be distinguished from disorders as fibro-adenoma, phyllodes tumor and fibrocystic abnormalities by imaging and from low-grade angiosarcoma by pathologic studies [6].

The most interesting patient of our series was a 40-year old woman, presenting with two palpable masses in her left breast since 6 weeks. On mammography an asymmetric

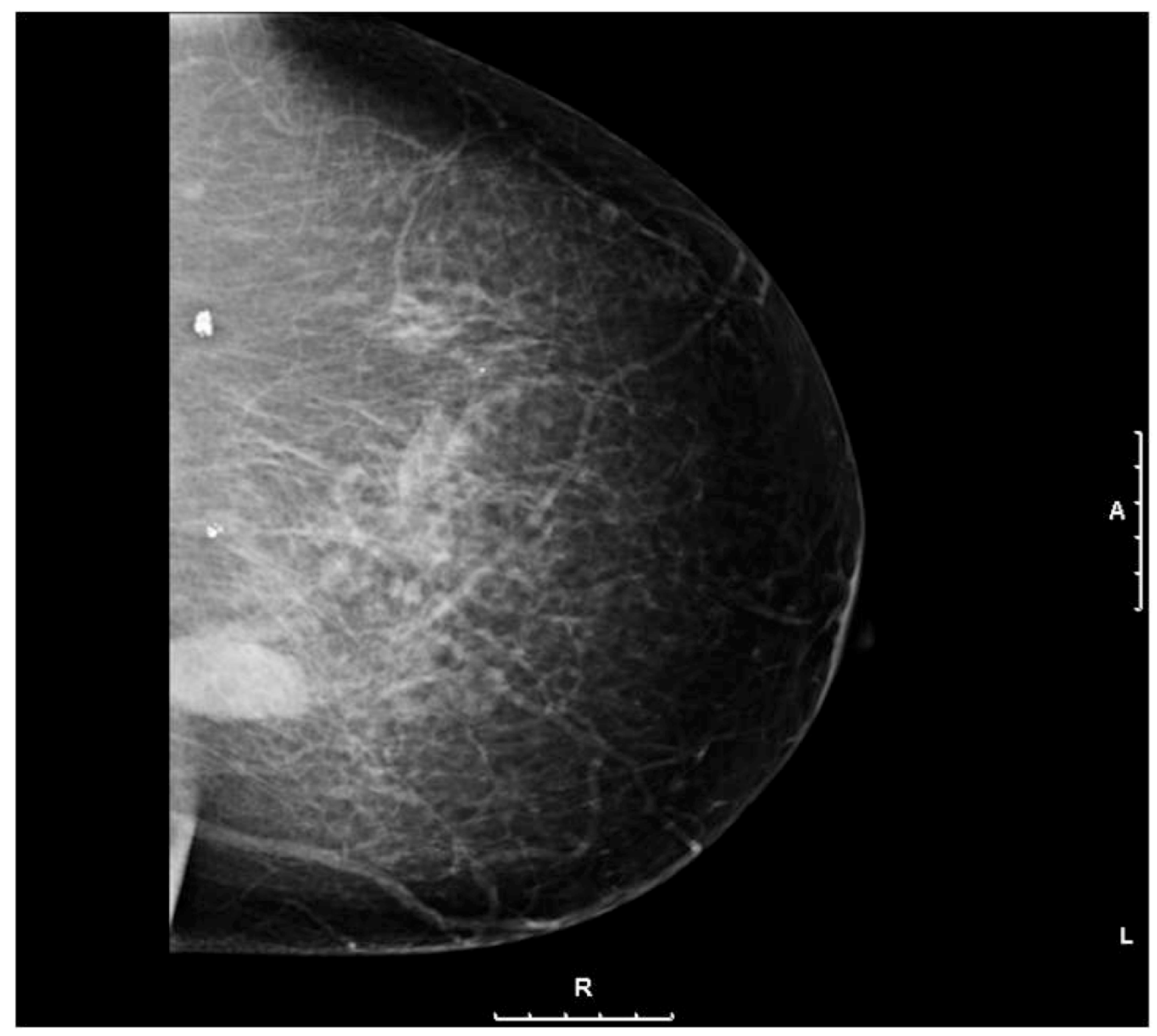

Fig. (2). Mammogram demonstrating a density. 


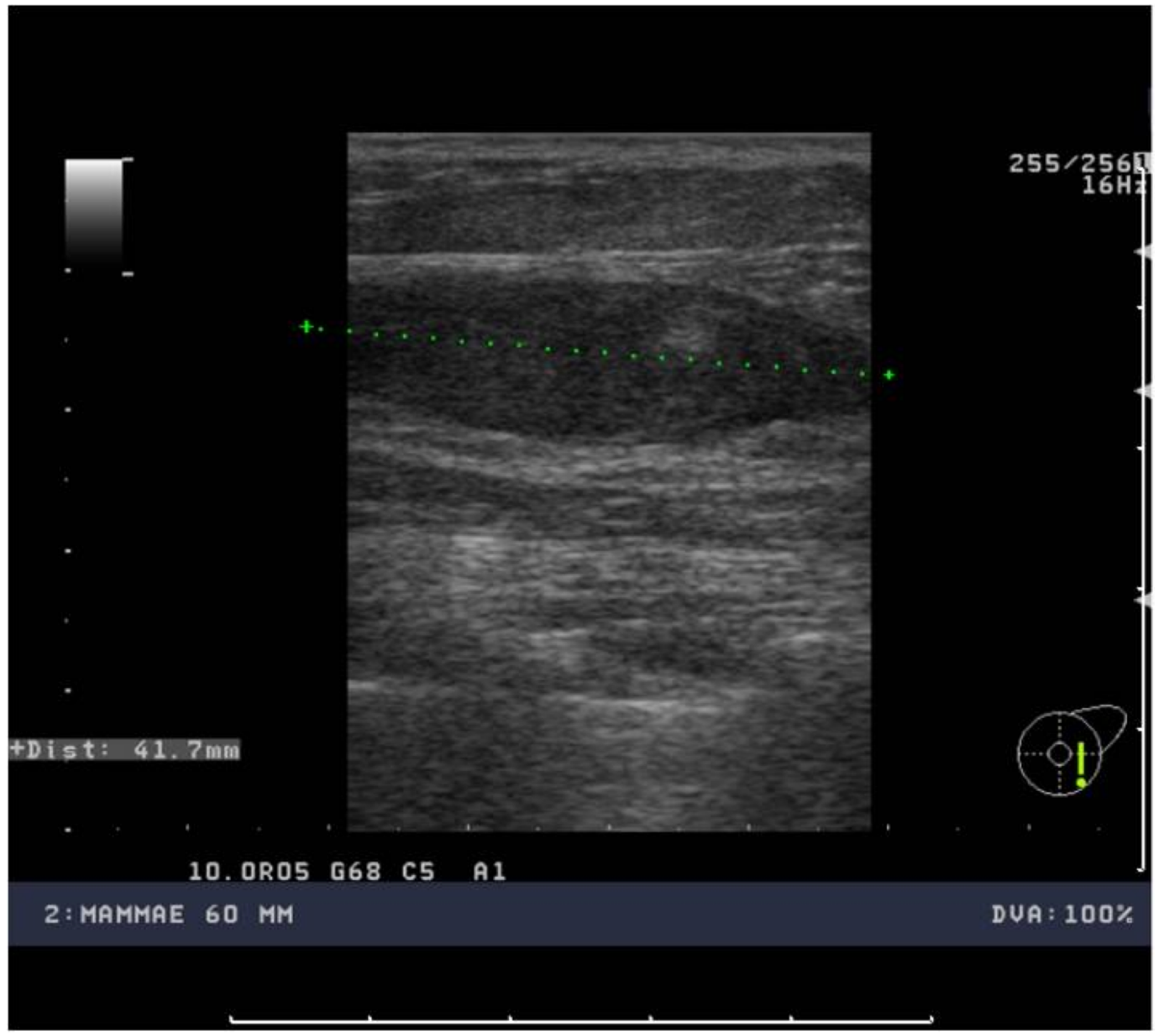

Fig. (3). Ultasound examination with an oval density oriented in parallel to the skin surface.

density was found, as US revealed an oval lesion of $1.8 \mathrm{~cm}$, imposing a fibroadenoma. Various core needle biopsies revealed PASH. On her own request, excision biopsy of the two lesions was performed, surprisingly revealing an invasive ductal carcinoma with accompanying DCIS grade III in one lesion, the other revealing fibroadenoma. She was then treated by mastectomy on her own request. Follow-up during 28 months revealed no abnormalities. We did not find any cases of ductal carcinoma accompanying PASH in the literature, although there are estimates that PASH can be identified in $25 \%$ of breast specimens [9]. This patient demonstrates the possibility of a sampling error. In our series we found three other patients in which sampling error can be considered (Table 2). Degnim et al. (2010) identified 579 patients with PASH in a cohort of 9065 patients with benign biopsies from the breast. Thirty four of these patients $(5.9 \%)$ developed breast cancer after a median follow-up of 18.1 years. In the group of non-PASH biopsies $8.8 \%$ of the patients developed breast cancer $(p<0.001)$. The authors concluded that patients with PASH seem to have a relative lower risk of developing breast cancer. However, patients with PASH were significantly younger [10].
Nowadays, excisional biopsy is no longer recommended if PASH is an incidental finding and if there is correspondence between clinical findings, imaging and core needle biopsy. However, in tumorous PASH excision is still recommended. We confirmed that expectant treatment is safe, although our follow up is relatively short. Our data also supports a policy in which excision is only offered to selected patients. Further indications for surgery may be mechanical complaints, pain or apprehension for an alternative (malignant) diagnosis [3, 11-13]. Follow-up is also not advised although regular screening is necessary for the life-time risk of breast cancer. There are also suggestions for watchfull waiting after the diagnosis of PASH $[11,12]$.

\section{CONCLUSION}

PASH is a benign lesion of the breast that may be treated expectantly in case of an incidental finding together with concordance between clinical findings and diagnostic imaging. In case of tumorous PASH, excision is advised. Core needle biopsy is obligatory; the use of FNA is doubtful. 


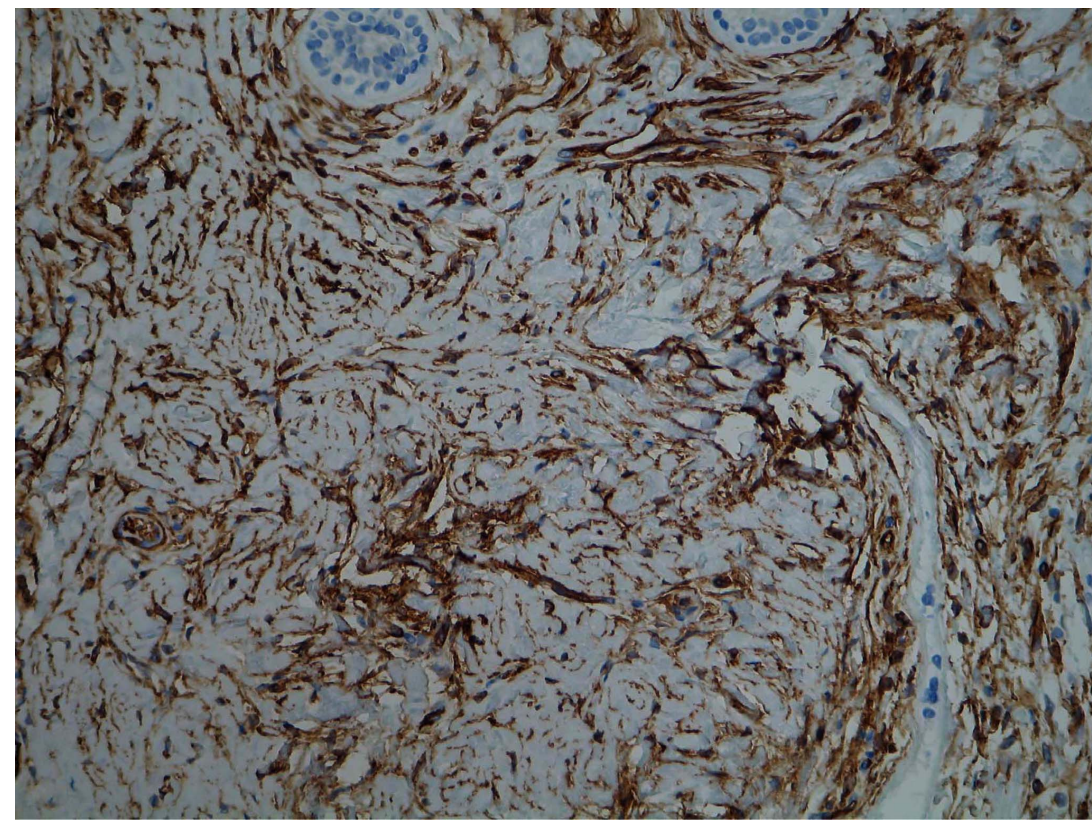

Fig. (4). Typical PASH lesion demonstrating pseudovascular spaces (H\&E staining, original magnification $200 \mathrm{x}$ ).

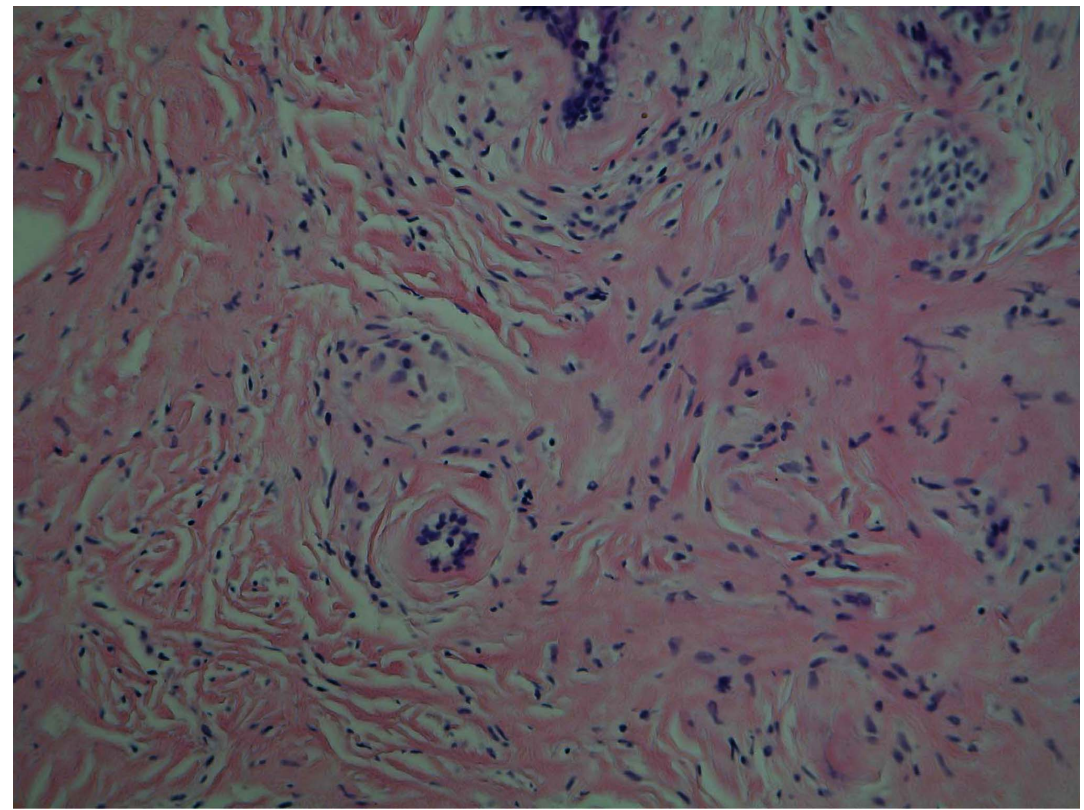

Fig. (5). Typical PASH lesion, pseudovascular spaces highlighted by CD34 staining (original magnification 200 x).

\section{CONFLICT OF INTEREST}

The authors do not report any conflict of interest

\section{REFERENCES}

[1] Vuitch MF, Rosen PP, Erlandson RA. Pseudoangiomatous hyperplasia of mammary stroma. Hum Pathol 1986; 17(2): 185-91.

[2] American College of Radiology. The ACR breast imaging reporting and data system (BI-RADS) [web source]. November 11, 2003. Available from: http://www.acr.org/SecondaryMainMenuCat egories/quality_safety/BIRADSAtlas.aspx

[3] Hargaden GC, Yeh ED, Georgian-Smith D, et al. Analysis of the mammographic and sonographic features of pseudoangiomatous stromal hyperplasia. AJR Am J Roentgenol 2008; 191(2): 359-63.

[4] Choi YJ, Ko EY, Kook S. Diagnosis of pseudoangiomatous stromal hyperplasia of the breast: ultrasonography findings and different biopsy methods. Yonsei Med J 2008; 49(5): 757-64.

[5] Levine PH, Nimeh D, Guth AA, Cangiarella JF. Aspiration biopsy of nodular pseudoangiomatous stromal hyperplasia of the breast: clinicopathologic correlates in 10 cases. Diagn Cytopathol 2005; 32(6): 345-50.

[6] Lui PC, Law BK, Chu WC, Pang LM, Tse GM. Fine-needle aspiration cytology of pseudoangiomatous stromal hyperplasia of the breast. Diagn Cytopathol 2004; 30(5): 353-5.

[7] Pruthi S, Reynolds C, Johnson RE, Gisvold JJ. Tamoxifen in the management of pseudoangiomatous stromal hyperplasia. Breast $\mathrm{J}$ 2001; 7(6): 434-9.

[8] Anderson C, Ricci A Jr, Pedersen CA, Cartun RW. Immunocytochemical analysis of estrogen and progesterone receptors in benign stromal lesions of the breast. Evidence for hormonal etiology in pseudoangiomatous hyperplasia of mammary stroma. Am J Surg Pathol 1991; 15(2): 145-9.

[9] Wieman SM, Landercasper J, Johnson JM, et al. Tumoral Pseudangiomatous Stromal Hyperplasia of the Breast. The American Surgeon 2008; 74: 1211-14.

[10] Degnim AC, Frost MH, Radisky DC, et al. Pseudoangiomatous Stromal Hyperplasia and Breast Cancer Risk. Ann Surg Oncol 2010; 17(12): 3269-77. 
[11] Polger MR, Denison CM, Lester S, Meyer JE. Pseudoangiomatous stromal hyperplasia: mammographic and sonographic appearances. AJR Am J Roentgenol 1996; 166(2): 349-52.

[12] Virk RK, Khan A. Pseudoangiomatous Stromal Hyperplasia An Overview. Arch Pathol Lab Med 2010; 134: 1070-74.
[13] Ferreira M, Albarracin CT, Resetkova E. Pseudoangiomatous stromal hyperplasia tumor: a clinical, radiologic and pathologic study of 26 cases. Mod Pathol 2008; 21(2): 201-7.

Received: May 10, 2011

Revised: June 15, 2011

Accepted: June 15, 2011

(C) Donk et al.; Licensee Bentham Open.

This is an open access article licensed under the terms of the Creative Commons Attribution Non-Commercial License (http://creativecommons.org/licenses/by-nc/ 3.0/) which permits unrestricted, non-commercial use, distribution and reproduction in any medium, provided the work is properly cited. 